УДК 547.992 .3

\title{
Study of Organosolv Lignins Using \\ Gel Permeation Chromatography \\ and Thermal Analysis
}

\author{
Yuriy N. Malyar*, Victor I. Sharypov, \\ Alexander S. Kazachenko and Alexander V. Levdansky \\ Institute of Chemistry and Chemical Technology SB RAS \\ FRC "Krasnoyarsk Scientific Center of the SB RAS" \\ 50/24 Akademgorodok, Krasnoyarsk, 660036, Russia
}

Received 20.12.2018, received in revised form 21.12.2018, accepted 28.01.2019

\begin{abstract}
Organosolv delignification of wood in the lower alcohols media is one of the promising methods for lignocellulosic biomass processing. The organosolv lignins obtained do not contain sulfur and ash, and therefore are a promising raw material for processing into valuable chemical products. To study the structure of lignins, a large range of physicochemical methods is used. In this review, attention is focused on the methods of studying the structure and properties of organosolv lignins, which have recently been greatly developed - gel permeation chromatography and thermal analysis methods. Examples of the use of these methods to study the molecular mass characteristics of organosolv lignins and their thermochemical properties are considered.
\end{abstract}

Keywords: organosolv lignins, structure, gel permeation chromatography, thermogravimetry, differential scanning calorimetry.

Citation: Malyar Yu.N., Sharypov V.I., Kazachenko A.S., Levdansky A.V. Study of organosolv lignins using gel permeation chromatography and thermal analysis, J. Sib. Fed. Univ. Chem., 2019, 12(1), 73-86. DOI: 10.17516/1998-2836-0109.

(c) Siberian Federal University. All rights reserved

* Corresponding author E-mail address: yumalyar@gmail.com 


\title{
Изучение органосольвентных лигнинов \\ с методами гель-проникающей хроматографии \\ и термического анализа
}

\author{
Ю.Н. Маляр, В.И. Шарыпов, \\ А.С. Казаченко, А.В. Левданский \\ Институт химии и химической технологии СО РАН \\ ФИЦ «Красноярский научный центр СО РАН» \\ Россия, 660036, Красноярск, Академгородок, 50/24
}

Органосольвентная делигнификаџия древесины в среде низиих спиртов является одним из перспективных методов переработки лигноцеллюлозной биомассы. Получаемые органосольвентные лигнины не содержат серы и золы и поэтому являются перспективным сырьем для переработки в ценные химические продукты. Для изучения строения лигнинов используется большой спектр физико-химических методов. В данном обзоре внимание акиентировано на методах исследования структуры и свойств органосольвентных лигнинов, получивших в последнее время большое развитие, - гель-проникающей хроматографии u термических методах анализа. Рассмотрены примеры использования этих методов для изучения молекулярно-массовых характеристик органосольвентных лигнинов и их термохимических свойств.

Ключевые слова: органосольвентные лигнины, структура, гель-проникающая хроматография, термогравиметрия, дифференциальная сканирующая калориметрия.

\section{Введение}

Лигнин представляет собой разветвленные аморфные полифенольные макромолекулы, синтезируемые в клеточной стенке путем сополимеризации от одного до трех прекурсоров, представляющих собой фенилпропановые производные (монолигнолы): конифериловый спирт, синапиловый спирт и п-кумариловый спирт. Процесс полимеризации начинается с ферментативного дегидрирования фенольных гидроксильных групп из монолигнолов с получением резонансно стабилизированных феноксирадикалов, которые могут случайным образом сочетаться друг с другом [1]. Объединение радикалов в различных сочетаниях создает различные типы межблочных связей, включая $\beta$-О-ариловый эфир ( $\beta-O-4), 4-О-5$ (бифениловый эфир) и углеродные связи, такие как 1,2-диарилпропан ( $\beta-1)$, резинол $(5-5)$, бифенил (5-5') и фенилкумаран $(\beta-5 / \alpha-\mathrm{O}-4)$.

Одним из перспективных направлений переработки низкомолекулярных органосольвентных лигнинов является их химическая модификация с сохранением первичной полимерной структуры. Изучено превращение лигнина в полимерные и другие высокомолекулярные молекулы путем удлинения лигниновой цепи с использованием пропиленоксида или этиленоксида. Удлинение и модифицирование полимерной цепи может существенно изменить физические свойства лигнина, превращая даже твердый крафт-лигнин в воск или вязкую жидкость [2]. 
Из-за сложной структуры лигнинов, полученных с использованием различных методов и условий, были разработаны новые аналитические подходы для описания их химических характеристик. Неоднородность лигнина обусловлена различиями в составе полимера, размере молекулярной цепи, сшивках и функциональных группах. При этом наиболее важными характеристиками, которые необходимо учитывать для любого потенциального промышленного применения лигнина, выступают чистота, молекулярная структура и термические свойства [3].

В данном обзоре рассмотрена возможность применения методов гель-проникающей хроматографии и термического анализа для изучения органосольвентных лигнинов.

\section{1. Гель-проникающая хроматография}

Одними из ключевых свойств для химической переработки и модификации лигнинов являются молекулярно-массовые характеристики. Среднечисловая молекулярная масса (Mn), среднемассовая молекулярная масса (Mw) и индекс полидисперсности (D) варьируются в широких пределах в зависимости от источника биомассы, условий предварительной обработки и метода выделения лигнина [4]. Гель-проникающая или эксклюзионная хроматография (ГПХ) один из наиболее распространенных методов, используемых для определения молекулярной массы лигнина [5]. Широкое применение ГПХ обусловлено коротким временем анализа (от 30 мин до 5 ч на образец); малыми навесками образцов; универсальностью по отношению к синтетическим и природным полимерам, а также широким диапазоном обнаружения молекулярных масс [6].

Основным отличием ГПХ от классической жидкостной хроматографии является применение колонок со сшитыми полимерами - гелями в качестве стационарной фазы. Однако механизм разделения в ГПХ опирается исключительно на размер молекул полимера в растворе, а не на любые химические взаимодействия между частицами и неподвижной фазой. Это отличие определяет свойства гелей - инертность, отсутствие ионных групп, контролируемый размер пор и в данном растворителе низкое сродство к подлежащим разделению веществам [7].

Из-за плохой растворимости лигнинов в различных средах перед выполнением ГПХанализа часто применяют дериватизацию путем силирования, метилирования, ацетилирования для улучшения растворимости $[8,9]$. Затем лигнины растворяют в соответствующем элюенте и вводят в пористую гелевую колонку, где происходит разделение молекул по размеру. Включение или исключение в порах геля определяется гидродинамическим радиусом молекул лигнина. Для определения относительного молекулярного размера и массы устанавливается зависимость между объемом элюирования и стандартом [8]. Для построения калибровочной кривой в ГПХ часто используются стандарты полистирола с полидисперсностью ниже 1.1 [10-12]. Но так как гидродинамические объемы лигнина и полистирола различаются, можно сказать, что ГПХ - относительный метод $[8,13]$. Тем не менее использование метода ГПХ в комбинации с такими методами, как вискозиметрия, МАЛДИ-ВП-МС [14] и низкоугловое светорассеяние [15] или многоугловое лазерное рассеяние света [16], обеспечивает получение более точных значений молекулярной массы, а также возможность определения размеров частиц, конформаций и вязкостных характеристик лигнинов [17]. 
Одно из традиционных применений ГПХ заключается в измерении степени деградации лигнина при различных методах делигнификации [18].

Glasser и др. успешно изучили молекулярные массы лигнинов из различных источников: лигнин Бьёркмана, кислотно-гидролизованных, пароактивированных, крафт- и органосольвентных. Было установлено, что ГПХ является универсальным методом для всех исследуемых типов лигнина. Более того, сравнение ГПХ с другими методами (МАЛДИ, парофазная осмометрия, светорассеяние и др.) подтверждает его надежность [6, 19-21].

ГПХ-анализ фракционирования крафт-лигнинов из различных видов древесины на более узкие фракции для коммерческого применения показал уменьшение полидисперсности с увеличением доли гексана в ацетоне [22]. В другом исследовании лигнины, выделенные из дикой и трансгенной люцерны, были исследованы с использованием ГПХ для определения различий в молекулярной массе в результате модификации. Трансгенные лигнины имели меньшие молекулярные массы, чем лигнины из дикой люцерны [23]. ГПХ также помог оценить молекулярные массы лигнина, выделенного из бесщелочных плат, изготовленных из термомеханической целлюлозы [22], остаточного ферментативного лигнина измельченной сосны [24], промышленного черного щелока [25], и влияние предварительной обработки разбавленной фосфорной кислотой и паром на лигнин сахарного тростника [26].

В последнее время ГПХ все чаще используют для описания молекулярно-массовых характеристик лигнинов в различных процессах. Установлены молекулярные массы эвкалиптового лигнина во время различных реакций щелочной делигнификации [27]; исследована эффективность органических оснований при делигнификации подкисленной и лиственной древесины [28], для оценки экстрагирования серной кислотой и перекисью водорода в щелочной среде [29], характеризация лигнинов для использования в производстве лигниновой пленки [30], для мониторинга ацетилированного лигнина Miscanthus $x$ giganteus до и после расщепления связей $\beta-O-4$ в процессе тиоацидолиза [31] и, наконец, для сравнения различных видов лигнинов, выделенных из ели Дугласа, белой ели, эвкалипта, красного дерева и сосны [32].

Многоугловое рассеяние лазерного света (MALLS) в сочетании с ГПХ позволило провести более подробный анализ лигнина и его агрегатов [33, 34]. Измеряемые интенсивности светорассеяния прямо пропорциональны молекулярной морфологии. В целом использование MALLS привело к значительному сокращению времени сбора данных и в дополнение к определению молекулярно-массового распределения также позволяет отслеживать различия в гидродинамических и стоксовых радиусах молекул. Авторы идентифицируют основное преимущество этого метода как его способность характеризовать крупные агрегаты лигнина, которые не были обнаружены с использованием рефрактометра или УФ-детекторов [34]. Отсутствие этих агрегатов в традиционном детектировании может быть неверно истолковано как уменьшение молекулярной массы лигнина, когда на самом деле эти агрегаты присутствуют. Этот метод был применен для изучения крафт-лигнина в различных растворителях [33].

На молекулярно-массовые характеристики лигнинов влияют методы их выделения: продолжительность, температура, состав смеси. В качестве сравнения в табл. 1 представлены молекулярно-массовые характеристики этаноллигнинов, полученных из различной биомассы и при разных условиях. Согласно приведенным данным, в среднем этаноллигнины имеют дисперсность 2-2,5 и молекулярную массу около 2кДа.

$$
-76-
$$


Таблица 1. Молекулярные массы этаноллигнинов из различных видов биомассы

Table 1. Molecular mass of ethanol lignins from various types of biomass

\begin{tabular}{|c|c|c|c|c|}
\hline № & Вид биомассы & $\mathrm{Mn}$ & Mw & $\mathrm{D}$ \\
\hline 1 & $\begin{array}{l}\text { Мискантус (Miscanthus giganteus) [35] } \\
\text { [36] }\end{array}$ & $\begin{array}{c}4690 \\
1170-1520\end{array}$ & $\begin{array}{c}7060 \\
2210-2720\end{array}$ & $\begin{array}{c}1,51 \\
1,64-2,03\end{array}$ \\
\hline 2 & Эвкалипт (Eucalyptus globulus) [37] & $1280-1360$ & $3990-4270$ & $2,81-3,16$ \\
\hline 3 & Эвкалипт (Eucalyptus pellita) [38] & $1102-1469$ & $1966-3185$ & $1,74-2,17$ \\
\hline 4 & Лириодендрон (Liriodendron tulipifera) [39] & $1155-1367$ & $2153-4276$ & $1,9-3,1$ \\
\hline 5 & Древесина бука [40] & - & 3433 & - \\
\hline 6 & Сосна лучистая (Pinus radiata) [41] & $97-713$ & $1653-10767$ & $5,74-27,58$ \\
\hline 7 & Рисовая шелуха [42] & 578 & 948 & 1,6 \\
\hline 8 & Багасса [42] & 691 & 1140 & 1,6 \\
\hline 9 & Солома пшеницы [42] & 517 & 1290 & 2,5 \\
\hline 10 & Измельченная древесина [42] & 1104 & 1436 & 1,3 \\
\hline 11 & $\begin{array}{l}\text { Немодифицированный автогидролизованный } \\
\text { органосольвентный этаноллигнин из пальмовых } \\
\text { листьев (AH EOL) [43], модифицированный } \\
\text { автогидролизованный органосольвентный } \\
\text { этаноллигнин (AHC EOL) }\end{array}$ & 4023 & 1581 & 2,54 \\
\hline 12 & Масличная пальма [44] & 2373 & 5151 & 2,17 \\
\hline 13 & Буддлея Давида (Buddleja davidii) [1] & $578-661$ & $2350-2740$ & $3,56-4,74$ \\
\hline 14 & Виноградные стебли [45] & 927 & 2984 & 3,22 \\
\hline 15 & Клен, тополь и береза [46] & 794 & 2975 & 3,7 \\
\hline 16 & Древесина бука [47] & 606 & 3428 & 5,66 \\
\hline 17 & Кора японского кедра [48] & $830-1120$ & $1826-2688$ & $2,2-2,8$ \\
\hline
\end{tabular}

\section{2. Термические методы исследования}

Термогравиметрический метод анализа (ТГА) регистрирует интегральную (ТГ) и дифференциальную (ДТГ) зависимость изменения массы анализируемого образца от температуры его нагревания с заданной скоростью. В соответствии с целями исследования термогравиметрический анализ проводят в потоке либо инертного газа, обычно аргона, либо в газах-окислителях - кислорода или воздуха [49]. Часто ТГА сочетают с методом дифференциальной сканирующей калориметрии (ДСК). В ДСК измеряется тепловой поток, необходимый для повышения с постоянной скоростью температуры анализируемого образца. ДТА и ДСК являются наиболее часто используемыми методами изучения термических свойств лигнина. ДСК обеспечивает более прямую информацию, чем ТГ и ДТА, относительно изменений энтальпии, связанных с фазовым переходом, происходящим при нагревании или охлаждении [50-52].

ТГА в инертной среде показывает, что лигнин наиболее термически устойчивый компонент биомассы. Он разлагается медленнее и в более широком температурном диапазоне (200$\left.500{ }^{\circ} \mathrm{C}\right)$, чем целлюлоза и гемицеллюлозы. Анализ литературных данных показывает, что ДТГ кривые потери массы образцов древесного лигнина включают только один интенсивный пик, 
согласно данным ТГ, с максимумом в интервале $230-440{ }^{\circ} \mathrm{C}$. При этом потеря массы образцов при конечной температуре пиролиза менялась от 41 до 73 мас.\% (табл. 2). На кривой ДТГ часто регистрируются минорные пики в области температур ниже и выше температуры максимума основного пика разложения лигнинов. Деструкция лигнина начинается при температуре 150$250{ }^{\circ} \mathrm{C}$ с гетеролитического расщепления связей $\beta-\mathrm{O}-4[51,53]$. По изменению скорости потери массы в данной области можно судить о количестве связей $\beta-\mathrm{O}-4$ в лигнинах. С повышением температуры до $250-350{ }^{\circ} \mathrm{C}$ достигается максимум скорости расщепления алкил-арильных простых эфирных связей $\alpha-\mathrm{O}-4$, а также происходит расщепление связей пропановых цепей $\mathrm{C}_{\beta}-\mathrm{C}_{\gamma}$ и $\mathrm{C} 1-\mathrm{C}_{\alpha}$. Параллельно реакциям расщепления этих связей при температуре $330{ }^{\circ} \mathrm{C}$ также проходят реакции рекомбинации свободных радикалов и вторичные гетеролитические реакции конденсации [54].

Важную информацию о термических превращениях лигнина можно получить, используя тандемную технологию сочетания ТГА с одновременным исследованием образующихся летучих продуктов методами ИК-спектроскопии (TГА - FTIR) и масс-спектрометрии (ТГА MSD).

Некоторые неконденсируемые газообразные компоненты с низкой молекулярной массой можно легко идентифицировать методом FTIR по их характеристическим полосам, таким как $\mathrm{CO}$ (при 2087 и $741 \mathrm{~cm}^{-1}$ ), $\mathrm{CO}_{2}$ (при $2310 \mathrm{~cm}^{-1}$ ), $\mathrm{CH}_{4}$ (при $2954 \mathrm{~cm}^{-1}$ ) и $\mathrm{H}_{2} \mathrm{O}$ (при $3599 \mathrm{~cm}^{-1}$ ). Образование $\mathrm{CH}_{4}$ в основном объясняется разложением метокси $\left(\mathrm{OCH}_{3}\right)$, метильных $\left(\mathrm{CH}_{3}\right)$ и метиленовых $\left(\mathrm{CH}_{2}\right)$ групп. $\mathrm{CO}_{2}$ образуется в результате реакции декарбоксилирования и декарбонилирования. $\mathrm{H}_{2} \mathrm{O}$ в основном образуется в реакции дегидроксилирования $[61,66]$.

В процессе термического превращения образцов лигнинов в условиях ТГА образуется широкий спектр летучих соединений. В отличие от хромато-масс-спектрометрии в TГA-MSD не используется хроматографическое разделение, поэтому идентификация индивидуальных веществ является сложной задачей. Одинаковый сигнал $\mathrm{m} / \mathrm{z}$ может соответствовать разным

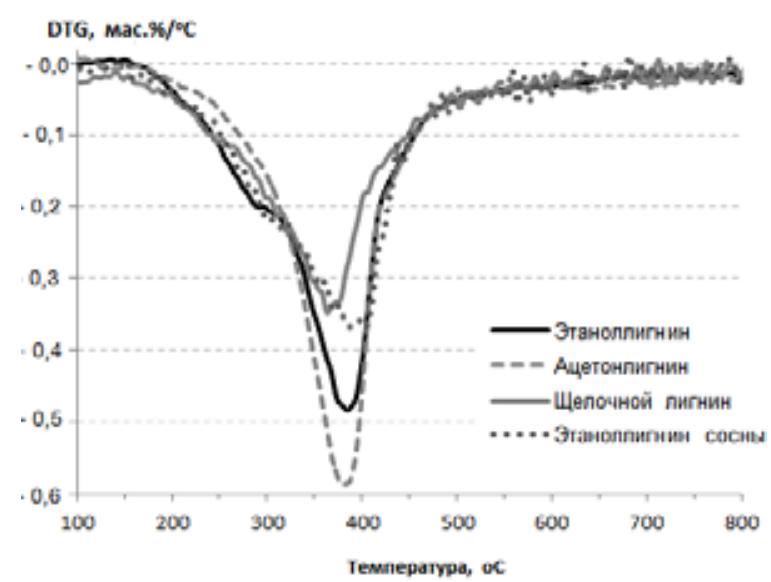

Рис. 1. Интегральные кривые потери массы лигнинов, выделенных из древесины осины и сосны разными методами $[56,57,59,60]$

Fig. 1. Integral weight loss curves of lignins isolated from aspen and pine wood by different methods $[56,57,59$, 60] 
Таблица 2. Показатели термического разложения древесных лигнинов, рассчитанные из результатов термогравиметрических исследований. Скорость подъема температуры $10{ }^{\circ} \mathrm{C} /$ мин в токе аргона

Table 2. Indicators of thermal decomposition of wood lignins, calculated from the results of thermogravimetric studies. The rate of temperature rise $10^{\circ} \mathrm{C} / \mathrm{min}$ under argon

\begin{tabular}{|c|c|c|c|c|c|}
\hline лигнин & \multirow{2}{*}{$\begin{array}{c}\text { ДТГ } \\
\text { максимум } \\
\text { основного } \\
\text { пика, }{ }^{\circ} \mathrm{C}\end{array}$} & \multirow{2}{*}{$\begin{array}{c}\text { Потеря } \\
\text { массы, } \\
\text { мас. } \%\end{array}$} & лигнин & \multirow{2}{*}{$\begin{array}{c}\text { ДТГ } \\
\text { максимум } \\
\text { основного } \\
\text { пика, }{ }^{\circ} \mathrm{C} \\
\end{array}$} & \multirow{2}{*}{$\begin{array}{c}\text { Потеря } \\
\text { массы, } \\
\text { мас.\% }\end{array}$} \\
\hline $\begin{array}{c}\text { Способ выделения / } \\
\text { тип древесины }\end{array}$ & & & $\begin{array}{c}\text { Способ выделения / } \\
\text { тип древесины }\end{array}$ & & \\
\hline Бьеркмана / бук [55] & 326 & 60,2 & Класона / осина [56] & 378 & 55,2 \\
\hline Бьеркмана / кедр [55] & 353 & 67,7 & Ацетонлигнин / осина [57] & 379 & 67,7 \\
\hline $\begin{array}{l}\text { Бьеркмана / Амурская липа } \\
\text { [58] }\end{array}$ & 342 & 41,0 & Этаноллигнин / осина [59] & 385 & 67,3 \\
\hline $\begin{array}{l}\text { Щелочной / Feihuang } \\
\text { Chemical Company [58] }\end{array}$ & 232 & 57,6 & Этаноллигнин / сосна [60] & 403 & 65,0 \\
\hline $\begin{array}{l}\text { Сульфонатный / Feihuang } \\
\text { Chemical Company [58] }\end{array}$ & 248 & 39,2 & $\begin{array}{l}\text { Lignoboost@ / хвойная } \\
\text { древесина [61] }\end{array}$ & 385 & 54,0 \\
\hline Класона / Сосна [62] & 438 & 53,6 & Класона / дуб [61] & 370 & 54,0 \\
\hline Класона / эвкалипт [62] & 389 & 51,5 & $\begin{array}{l}\text { Органосольвентный / } \\
\text { береза [61] }\end{array}$ & 380 & 54,0 \\
\hline $\begin{array}{l}\text { Крафт / Sigma-Aldrich Co, } \\
\text { Ltd [63] }\end{array}$ & 325 & 56,0 & $\begin{array}{l}\text { Бьеркмана / ксилема } \\
\text { тополя [64] }\end{array}$ & $\begin{array}{c}\text { Два } \\
\text { основных } \\
\text { максимума } \\
290,6 \text { и 359,4 }\end{array}$ & 73,4 \\
\hline Щелочной / осина [56] & 364 & 60,2 & $\begin{array}{l}\text { Этаноллигнин / ксилема } \\
\text { тополя [64] }\end{array}$ & 352 & 61,8 \\
\hline $\begin{array}{l}\text { Уксуснокислотный / осина } \\
\text { [56] }\end{array}$ & 407 & 61,4 & *EMAL / сосна [65] & 358 & 62,0 \\
\hline
\end{tabular}

ионам различных веществ. Например, $\mathrm{m} / \mathrm{z} 16$ можно отнести к $\mathrm{O}^{+}$в воде и $\mathrm{CH}_{4}{ }^{+}$в метане, $\mathrm{m} / \mathrm{z} 17$ к $\mathrm{OH}^{+}$и $\mathrm{NH}_{3}{ }^{+}, \mathrm{m} / \mathrm{z} 28$ к $\mathrm{N}_{2}{ }^{+}, \mathrm{CO}^{+}$и $\mathrm{C}_{2} \mathrm{H}_{4}{ }^{+}, \mathrm{m} / \mathrm{z} 44$ к $\mathrm{CO}_{2}{ }^{+}$и $\mathrm{C}_{2} \mathrm{H}_{4} \mathrm{OH}^{+}$. Кроме того, одинаковый ион может образовываться из разных веществ. Бензилион $\mathrm{C}_{6} \mathrm{H}_{5} \mathrm{CH}_{2}{ }^{+} \mathrm{c} \mathrm{m} / \mathrm{z} 91$ может образовываться при деструкции алкилбензолов, как и при деструкции алкилпроизводных простых фенолов или метоксифенолов [65, 66].

Вода и диоксид углерода являются основными продуктами деструкции лигнинов [67]. Образование воды при ТГА лигнина может происходить при деструкции нескольких типов О-содержащих функциональных групп. Две стадии (150-300 и 300-500 $\left.{ }^{\circ} \mathrm{C}\right)$ наблюдались для лигнина Lignoboost ${ }^{\circledR}$ из древесины хвойных пород. В этом же исследовании только одна основная стадия выше $250^{\circ} \mathrm{C}$ была установлена для лигнина из лиственных пород деревьев - дуба и березы [61]. Две стадии выделения воды с максимумами при 210 и $440{ }^{\circ} \mathrm{C}$ обнаружены при исследовании древесины сосны [65]. Динамика изменения сигнала m/z 44 показала, что выделение $\mathrm{CO}_{2}$ происходит в две стадии при более высоких температурах 395 и $515^{\circ} \mathrm{C}$, чем стадии выделения воды. Образование $\mathrm{CO}_{2}$ при разложении лигнина березы происходит в одну стадию, максимум пика которой $430^{\circ} \mathrm{C}$ практически совпадает со стадией выделения воды. Фенол (m/z 94) является одним из важных продуктов термического разложения лигнинов. В процессе TГА лигнина EMAL из древесины сосны образование фенола начинается при температуре 
ниже $300{ }^{\circ} \mathrm{C}$. Этот факт, по мнению авторов, указывает на образование фенола в результате разрыва $\beta-\mathrm{O}-4$ эфирной связи в лигнине. Максимум пика выхода фенола наблюдается при $430{ }^{\circ} \mathrm{C}[65]$.

ДСК обеспечивает прямую информацию об изменении энтальпии, связанном с фазовым переходом, происходящим при нагревании или охлаждении [50-52]. Наиболее важными параметрами, которые можно установить путем ДТГ, являются температура стеклования (Tg) и изменение теплоемкости $\left(\mathrm{C}_{\mathrm{p}}\right)$. Результаты исследования ДСК лигнинов из разных пород деревьев показали, что самая низкая теплоемкость характерна для лигнина из древесины смешанных лиственных пород $\left(0,009\right.$ Дж $\left./ \Gamma^{\circ} \mathrm{C}\right)$, а самая высокая - для лигнина сосны $\left(0,417\right.$ Дж $\left./ \Gamma^{\circ} \mathrm{C}\right)$. Самая низкая температура стеклования $\left(\mathrm{T}_{\mathrm{g}}\right)$ получена для лигнина осины $-89,9^{\circ} \mathrm{C}$, максимальное значение $\mathrm{Tg}=180,6^{\circ} \mathrm{C}$ установлено для лигнина из смешанных лиственных пород [68]. Методы выделения лигнина также оказывают существенное влияние на показатели ДСК. Крафт-лигнины имеют более высокие значения $\mathrm{T}_{\mathrm{g}}$, тогда как органосольвентные лигнины - более низкие [69]. На величину $\mathrm{T}_{\mathrm{g}}$ также могут оказывать влияние параметры обработки, о чем свидетельствует широкий диапазон температур для лигнина из смешанных лиственных пород. Изменения значений $\mathrm{T}_{\mathrm{g}}$ лигнинов, а также изменения теплоемкости иллюстрируют изменения их химических структур, которые могут быть сопоставлены с изменениями гибкости полимерных цепей, степенью полимеризации и количеством примесей [70, 71].

Кинетические исследования лигнина, представленные в литературе, основаны на различных моделях его деструкции [53, 72-74]. Обычно термическое разложение лигнина описывается реакцией первого порядка с энергией активации от 54,34 до 79,42 кДж/моль в температурном диапазоне $244-309{ }^{\circ} \mathrm{C}[53,75]$. В температурном диапазоне $327-1167{ }^{\circ} \mathrm{C}$ происходит увеличение

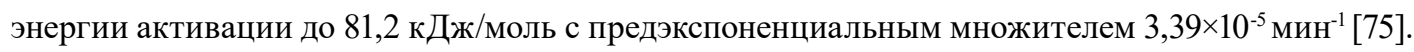

Предложена более сложная кинетическая модель, предполагающая, что лигнин образуется «фракциями», которые разлагаются только выше характеристических температур [76, 77]. Согласно этой модели энергия активации линейно увеличивается от 72,4 до 174 кДж/моль в интервале температур 200-600 ${ }^{\circ} \mathrm{C}$. Методами ТГ и ДСК исследовали сложные схемы параллельных, последовательных и конкурентных реакций псевдопервого порядка разложения лигнинов Бьеркмана. Энергии активации данных реакций составили 34-65 кДж/моль и предэкспоненциальный множитель $10^{0,3}-10^{3}$ [78]. Кинетические исследования пиролиза органосольвентных и крафт-лигнинов в температурном диапазоне $170-630{ }^{\circ} \mathrm{C}$ в стационарном реакторе показали более низкие энергии активации 23-79 кДж/моль и 17-89 кДж/моль соответственно, что, вероятно, связано со значительными эффектами массопереноса и теплопередачи. Энергии активации для органосольвентных и крафт-лигнинов составили 129-361 и 80-158 кДж / моль с предэкспоненциальным множителем 6,2 × $10^{11}$-9,3 $\times 10^{22}$ и 3,3 $\times 10^{7}-1,8 \times 10^{9}$ при скорости нагревания $5-15{ }^{\circ} \mathrm{C}$ до конечной температуры $800{ }^{\circ} \mathrm{C}[52]$.

Исследование выполнено при финансовой поддержке Российского фонда фундаментальных исследований, Правительства Красноярского края, Красноярского краевого фонда науки в рамках научного проекта: «Модификация древесных лигнинов с получением перспективных фармакологически активных и светочувствительных водорастворимых полимеров" № 18-43-243016 р_ммол_а. 


\section{Список литературы}

1. Hallac B.B., Pu Y., Ragauskas A.J. Chemical transformations of Buddleja davidii lignin during ethanol organosolv pretreatment. Energy and Fuels 2010. Vol. 24(4), P. 2723-2732.

2. Holladay J.E., White J.F., Bozell J.J., Johnson D. Top Value-Added Chemicals from Biomass Volume II - Results of Screening for Potential Candidates from Biorefinery Lignin. Pacific Northwest National Laboratory 2007. Vol. II(October). P. 87.

3. Fang Z., Smith R.L. Production of Biofuels and Chemicals from Lignin. / ed. Fang Z., Smith R.L. Singapore: Springer Singapore, 2016.

4. Tolbert A., Akinosho H., Khunsupat R., Naskar A.K., Ragauskas A.J. Characterization and analysis of the molecular weight of lignin for biorefining studies. Biofuels, Bioproducts and Biorefining 2014. Vol. 8(6), P. 836-856.

5. Lupoi J.S., Singh S., Parthasarathi R., Simmons B.A., Henry R.J. Recent innovations in analytical methods for the qualitative and quantitative assessment of lignin. Renewable and Sustainable Energy Reviews Elsevier, 2015. Vol. 49, P. 871-906.

6. Glasser W.G., Barnett C.A., Muller P.C., Sarkanen K.V. The chemistry of several novel bioconversion lignins. Journal of Agricultural and Food Chemistry 1983. Vol. 31(5), P. 921-930.

7. Determann H. Gel Chromatography, Gel Filtration, Gel Permeation, Molecular Sieves: A Laboratory Handbook. Springer-Verlag Berlin Heidelberg, 1969. 202 p.

8. Brunow G., Brunow P.G. Methods to Reveal the Structure of Lignin. Lignin, Humic Substances and Coal / ed. Steinbüchel A., Hofrichter M. Weinheim, Germany: Wiley-VCH Verlag GmbH \& Co. KGaA, 2005. P. 89-99.

9. Asikkala J., Tamminen T., Argyropoulos D.S. Accurate and Reproducible Determination of Lignin Molar Mass by Acetobromination. Journal of Agricultural and Food Chemistry 2012. Vol. 60(36), P. 8968-8973.

10. Faix O., Beinhoff O. Short Note Improved Calibration of High-Performance Size-Exclusion Chromatography of Lignins Using Ligninlike Model Compounds. Holzforschung 1992. Vol. 46(4), P. 355-356.

11. Botaro V.R., Curvelo A.A. da S. Monodisperse lignin fractions as standards in size-exclusion analysis. Journal of Chromatography A 2009. Vol. 1216(18), P. 3802-3806.

12. Baumberger S., Abaecherli A., Fasching M., Gellerstedt G., Gosselink R., Hortling B., Li J., Saake B., et al. Molar mass determination of lignins by size-exclusion chromatography: towards standardisation of the method. Holzforschung 2007. Vol. 61(4).

13. Himmel M.E., Tatsumoto K., Grohmann K., Johnson D.K., Chum H.L. Molecular weight distribution of aspen lignins from conventional gel permeation chromatography, universal calibration and sedimentation equilibrium. Journal of Chromatography A 1990. Vol. 498(C), P. 93-104.

14. Hanton S.D. Mass Spectrometry of Polymers and Polymer Surfaces. Chemical Reviews 2001. Vol. 101(2), P. 527-570.

15. Siochi E.J., Ward T.C., Haney M.A., Mahn B. The absolute molecular weight distribution of hydroxypropylated lignins. Macromolecules 1990. Vol. 23(5), P. 1420-1429.

16. Mikame K., Funaoka M. Polymer Structure of Lignophenol II - Comparison of Molecular Morphology of Lignophenol and Conventional Lignins. Polymer Journal 2006. Vol. 38(6), P. 592-596. 
17. Hiemenz P.C., Lodge T.P. Polymer Chemistry, Second Edition. CRC Press, 2007. 608 p.

18. Wu M., Pang J., Lu F., Zhang X., Che L., Xu F., Sun R. Application of new expansion pretreatment method on agricultural waste. Part I: Influence of pretreatment on the properties of lignin. Industrial Crops and Products 2013. Vol. 50 P. 887-895.

19. Zhu H., Yalcin T., Li L. Analysis of the accuracy of determining average molecular weights of narrow polydispersity polymers by matrix-assisted laser desorption ionization time-offlight mass spectrometry. Journal of the American Society for Mass Spectrometry 1998. Vol. 9(4), P. 275-281.

20. Froment P., Pla F. Determinations of Average Molecular Weights and Molecular Weight Distributions of Lignin. 1989. P. 134-143.

21. Chen F., Li J. Aqueous Gel Permeation Chromatographic Methods for Technical Lignins. Journal of Wood Chemistry and Technology 2000. Vol. 20(3), P. 265-276.

22. Sun Y.-C., Lin Z., Peng W.-X., Yuan T.-Q., Xu F., Wu Y.-Q., Yang J., Wang Y.-S., et al. Chemical Changes of Raw Materials and Manufactured Binderless Boards during Hot Pressing: Lignin Isolation and Characterization. BioResources 2014. Vol. 9(1).

23. Ziebell A., Gracom K., Katahira R., Chen F., Pu Y., Ragauskas A., Dixon R.A., Davis M. Increase in 4-Coumaryl Alcohol Units during Lignification in Alfalfa (Medicago sativa) Alters the Extractability and Molecular Weight of Lignin. Journal of Biological Chemistry 2010. Vol. 285(50), P. 38961-38968.

24. Holtman K.M., Chang H., Kadla J.F. An NMR Comparison of the Whole Lignin from Milled Wood, MWL, and REL Dissolved by the DMSO/NMI Procedure. Journal of Wood Chemistry and Technology 2007. Vol. 27(3-4), P. 179-200.

25. Hu J., Shen D., Xiao R., Wu S., Zhang H. Free-Radical Analysis on Thermochemical Transformation of Lignin to Phenolic Compounds. Energy \& Fuels 2013. Vol. 27(1), P. 285293.

26. Zeng J., Tong Z., Wang L., Zhu J.Y., Ingram L. Isolation and structural characterization of sugarcane bagasse lignin after dilute phosphoric acid plus steam explosion pretreatment and its effect on cellulose hydrolysis. Bioresource Technology 2014. Vol. 154, P. 274-281.

27. Prinsen P., Rencoret J., Gutiérrez A., Liitiä T., Tamminen T., Colodette J.L., Berbis M.Á., Jiménez-Barbero J., et al. Modification of the Lignin Structure during Alkaline Delignification of Eucalyptus Wood by Kraft, Soda-AQ, and Soda-O 2 Cooking. Industrial \& Engineering Chemistry Research 2013. Vol. 52(45), P. 15702-15712.

28. Xia G.-G., Chen B., Zhang R., Zhang Z.C. Catalytic hydrolytic cleavage and oxy-cleavage of lignin linkages. Journal of Molecular Catalysis A: Chemical 2014. Vol. 388-389 P. 35-40.

29. Savy D., Piccolo A. Physical-chemical characteristics of lignins separated from biomasses for second-generation ethanol. Biomass and Bioenergy 2014. Vol. 62, P. 58-67.

30. Pereira A.A., Martins G.F., Antunes P.A., Conrrado R., Pasquini D., Job A.E., Curvelo A.A.S., Ferreira M., et al. Lignin from Sugar Cane Bagasse: Extraction, Fabrication of Nanostructured Films, and Application. Langmuir 2007. Vol. 23(12), P. 6652-6659.

31. Villaverde J.J., Li J., Ek M., Ligero P., de Vega A. Native Lignin Structure of Miscanthus x giganteus and Its Changes during Acetic and Formic Acid Fractionation. Journal of Agricultural and Food Chemistry 2009. Vol. 57(14), P. 6262-6270. 
32. Guerra A., Filpponen I., Lucia L.A., Argyropoulos D.S. Comparative Evaluation of Three Lignin Isolation Protocols for Various Wood Species. Journal of Agricultural and Food Chemistry 2006. Vol. 54(26), P. 9696-9705.

33. Gidh A.V., Decker S.R., See C.H., Himmel M.E., Williford C.W. Characterization of lignin using multi-angle laser light scattering and atomic force microscopy. Analytica Chimica Acta 2006. Vol. 555(2), P. 250-258.

34. Gidh A. V., Decker S.R., Vinzant T.B., Himmel M.E., Williford C. Determination of lignin by size exclusion chromatography using multi angle laser light scattering. Journal of Chromatography A 2006. Vol. 1114(1), P. 102-110.

35. Hage R. El, Brosse N., Chrusciel L., Sanchez C., Sannigrahi P., Ragauskas A. Characterization of milled wood lignin and ethanol organosolv lignin from miscanthus. Polymer Degradation and Stability Elsevier Ltd, 2009. Vol. 94(10), P. 1632-1638.

36. Bauer S., Sorek H., Mitchell V.D., Ibáñez A.B., Wemmer D.E. Characterization of Miscanthus giganteus lignin isolated by ethanol organosolv process under reflux condition. Journal of Agricultural and Food Chemistry 2012. Vol. 60(33), P. 8203-8212.

37. Yáñez-S M., Matsuhiro B., Nuñez C., Pan S., Hubbell C.A., Sannigrahi P., Ragauskas A.J. Physicochemical characterization of ethanol organosolv lignin (EOL) from Eucalyptus globulus: Effect of extraction conditions on the molecular structure. Polymer Degradation and Stability 2014. Vol. 110 P. 184-194.

38. Choi J.-H., Jang S.-K., Kim J.-H., Park S.-Y., Kim J.-C., Jeong H., Kim H.-Y., Choi I.-G. Simultaneous production of glucose, furfural, and ethanol organosolv lignin for total utilization of high recalcitrant biomass by organosolv pretreatment. Renewable Energy Elsevier Ltd, 2019. Vol. 130, P. 952-960.

39. Jang S.-K., Kim H.-Y., Jeong H.-S., Kim J.-Y., Yeo H., Choi I.-G. Effect of ethanol organosolv pretreatment factors on enzymatic digestibility and ethanol organosolv lignin structure from Liriodendron tulipifera in specific combined severity factors. Renewable Energy Elsevier Ltd, 2016. Vol. 87, P. 599-606.

40. Lê H.Q., Zaitseva A., Pokki J.P., Ståhl M., Alopaeus V., Sixta H. Solubility of Organosolv Lignin in $\gamma$-Valerolactone/Water Binary Mixtures. ChemSusChem 2016. Vol. 9(20), P. 2939-2947.

41. Domínguez J.C., Santos T.M., Rigual V., Oliet M., Alonso M.V., Rodriguez F. Thermal stability, degradation kinetics, and molecular weight of organosolv lignins from Pinus radiata. Industrial Crops and Products Elsevier, 2018. Vol. 111(October), P. 889-898.

42. Singh S.K., Dhepe P.L. Experimental evidences for existence of varying moieties and functional groups in assorted crop waste derived organosolv lignins. Industrial Crops and Products Elsevier, 2018. Vol. 119(October 2017), P. 144-151.

43. Sa'don N.A., Rahim A.A., Ibrahim M.N.M., Brosse N., Hussin M.H. Modification of oil palm fronds lignin by incorporation of $\mathrm{m}$-cresol for improving structural and antioxidant properties. International Journal of Biological Macromolecules Elsevier B.V., 2017. Vol. 104, P. 251-260.

44. Hussin M.H., Rahim A.A., Mohamad Ibrahim M.N., Perrin D., Yemloul M., Brosse N. Impact of catalytic oil palm fronds (OPF) pulping on organosolv lignin properties. Polymer Degradation and Stability 2014. Vol. 109, P. 33-39. 
45. Amendola D., De Faveri D.M., Egües I., Serrano L., Labidi J., Spigno G. Autohydrolysis and organosolv process for recovery of hemicelluloses, phenolic compounds and lignin from grape stalks. Bioresource Technology Elsevier Ltd, 2012. Vol. 107, P. 267-274.

46. Liu Z., Fatehi P., Jahan M.S., Ni Y. Separation of lignocellulosic materials by combined processes of pre-hydrolysis and ethanol extraction. Bioresource Technology Elsevier Ltd, 2011. Vol. 102(2), P. 1264-1269.

47. Hashmi S.F., Meriö-Talvio H., Hakonen K.J., Ruuttunen K., Sixta H. Hydrothermolysis of organosolv lignin for the production of bio-oil rich in monoaromatic phenolic compounds. Fuel Processing Technology Elsevier, 2017. Vol. 168(September), P. 74-83.

48. Watanabe M., Kanaguri Y., Smith R.L. Hydrothermal separation of lignin from bark of Japanese cedar. Journal of Supercritical Fluids Elsevier, 2018. Vol. 133(September 2017), P. 696-703.

49. Kanaujia P.K., Sharma Y.K., Garg M.O., Tripathi D., Singh R. Review of analytical strategies in the production and upgrading of bio-oils derived from lignocellulosic biomass. Journal of Analytical and Applied Pyrolysis 2014. Vol. 105(1), P. 55-74.

50. Lin S.Y., Dence C.W. Method in lignin chemistry. Methods in Lignin Chemistry 1992. 578 p.

51. Stark N.M., Yelle D.J., Agarwal U.P. Techniques for Characterizing Lignin. Lignin in Polymer Composites Elsevier Inc., 2015. 49-66 p.

52. Ferdous D., Dalai A.K., Bej S.K., Thring R.W. Pyrolysis of lignins: Experimental and kinetics studies. Energy and Fuels 2002. Vol. 16(6), P. 1405-1412.

53. Brebu M., Vasile C. Thermal degradation of lignin - a review. Cellulose Chemistry \& Technology 2010. Vol. 44(9), P. 353-363.

54. Awal A., Sain M. Spectroscopic studies and evaluation of thermorheological properties of softwood and hardwood lignin. Journal of Applied Polymer Science 2011. Vol. 122(2), P. 956-963.

55. Asmadi M., Kawamoto H., Saka S. Gas- and solid/liquid-phase reactions during pyrolysis of softwood and hardwood lignins. Journal of Analytical and Applied Pyrolysis 2011. Vol. 92(2), P. 417425 .

56. Sharypov V.I., Grishechko L.I., Baryshnikov S.V., Miroshnikova A.V., Kuznetsov B.N. Investigation of Thermal Decomposition of Lignin Samples Isolated from Aspen Wood by Various Methods. Journal of Siberian Federal University. Chemistry 2011. Vol. 3(4), P. 221-231.

57. Шарыпов В.И., Береговцова Н.Г., Барышников С.В., Мирошникова А.В., Яковлев В.А., Кузнецов Б.Н. Термическая конверсия ацетонлигнина в сверхкритическом бутаноле в присутствии никельсодержащих катализаторов. Нижний Новгород, 2017. 22-26 p. [Sharypov V.I., Beregovtsova N.G., Baryshnikov S.V., Miroshnikova A.V., Yakovlev V.A., Kuznetsov B.N. Thermal conversion of acetone lignin in supercritical butanol in the presence of nickel-containing catalysts. Nizhny Novgorod, 2017. P. 22-26 (In Russ.)]

58. Lin X., Sui S., Tan S., Pittman C., Sun J., Zhang Z. Fast Pyrolysis of Four Lignins from Different Isolation Processes Using Py-GC/MS. Energies 2015. Vol. 8(6), P. 5107-5121.

59. Sharypov V.I., Beregovtsova N.G., Baryshnikov S.V., Miroshnikova A.V., Kuznetsov B.N. Study of Composition and Thermal Properties of Ethanollignin Isolated from Aspen-Wood. Journal of Siberian Federal University. Chemistry 2016. Vol. 9(3), P. 296-307.

60. Sharypov V.I., Beregovtsova N.G., Baryshnikov S.V., Miroshnikova A.V., Lavrenov A.V., Kuznetsov B.N. Conversion of Ethanol-Lignin from Pine Wood in a Supercritical Ethanol in the 
Presence of Borate-Containing Alumina Catalysts. Journal of Siberian Federal University. Chemistry 2018. Vol. 11(1), P. 81-92.

61. Brebu M., Tamminen T., Spiridon I. Thermal degradation of various lignins by TG-MS/FTIR and Py-GC-MS. Journal of Analytical and Applied Pyrolysis 2013. Vol. 104, P. 531-539.

62. Poletto M. Assessment of the thermal behavior of lignins from softwood and hardwood species. Maderas. Ciencia y tecnología 2017. (ahead), P. 63-74.

63. Ma Z., Sun Q., Ye J., Yao Q., Zhao C. Study on the thermal degradation behaviors and kinetics of alkali lignin for production of phenolic-rich bio-oil using TGA-FTIR and Py-GC/MS. Journal of Analytical and Applied Pyrolysis 2016. Vol. 117, P. 116-124.

64. Kim J.-Y., Oh S., Hwang H., Kim U.-J., Choi J.W. Structural features and thermal degradation properties of various lignin macromolecules obtained from poplar wood (Populus albaglandulosa). Polymer Degradation and Stability 2013. Vol. 98(9), P. 1671-1678.

65. Huang Y., Liu H., Yuan H., Zhuang X., Yuan S., Yin X., Wu C. Association of chemical structure and thermal degradation of lignins from crop straw and softwood. Journal of Analytical and Applied Pyrolysis 2018. Vol. 134, P. 25-34.

66. Ma Z., Sun Q., Ye J., Yao Q., Zhao C. Study on the thermal degradation behaviors and kinetics of alkali lignin for production of phenolic-rich bio-oil using TGA-FTIR and Py-GC/MS. Journal of Analytical and Applied Pyrolysis Elsevier B.V., 2016. Vol. 117, P. 116-124.

67. Brebu M., Spiridon I. Co-pyrolysis of LignoBoost ${ }^{\circledR}$ lignin with synthetic polymers. Polymer Degradation and Stability 2012. Vol. 97(11), P. 2104-2109.

68. Sammons R.J., Harper D.P., Labbé N., Bozell J.J., Elder T., Rials T.G. Characterization of organosolv lignins using thermal and FT-IR spectroscopic analysis. BioResources 2013. Vol. 8(2), P. 2752-2767.

69. Tejado A., Peña C., Labidi J., Echeverria J.M., Mondragon I. Physico-chemical characterization of lignins from different sources for use in phenol-formaldehyde resin synthesis. Bioresource Technology 2007. Vol. 98(8), P. 1655-1663.

70. Khan M.A., Ashraf S.M. Studies on thermal characterization of lignin: Substituted phenol formaldehyde resin as wood adhesives. Journal of Thermal Analysis and Calorimetry 2007. Vol. 89(3), P. 993-1000.

71. Kubo S., Kadla J.F. Poly ( Ethylene Oxide )/ Organosolv Lignin Blends: Relationship between Thermal Properties, Chemical Structure, and Blend Behavior. Society 2004. P. 6904-6911.

72. Koufopanos C.A., Lucchesi A., Maschio G. Kinetic modelling of the pyrolysis of biomass and biomass components. The Canadian Journal of Chemical Engineering 1989. Vol. 67(1), P. 75-84.

73. Caballero J., Font R., Marcilla A. Kinetic study of the secondary thermal decomposition of Kraft lignin. Journal of Analytical and Applied Pyrolysis 1996. Vol. 38(1-2), P. 131-152.

74. Caballero J.A., Font R., Marcilla A. Pyrolysis of Kraft lignin: yields and correlations. Journal of Analytical and Applied Pyrolysis 1997. Vol. 39(2), P. 161-183.

75. Nunn T.R., Howard J.B., Longwell J.P., Peters W.A. Product compositions and kinetics in the rapid pyrolysis of milled wood lignin. Industrial \& Engineering Chemistry Process Design and Development 1985. Vol. 24(3), P. 844-852.

76. Caballero J.A., Font R., Marcilla A., Conesa J.A. New kinetic model for thermal decomposition of heterogeneous materials. Industrial \& Engineering Chemistry Research 1995. Vol. 34(3), P. 806-812. 
77. Caballero J.A., Font R., Marcilla A. Study of the primary pyrolysis of Kraft lignin at high heating rates: yields and kinetics. Journal of Analytical and Applied Pyrolysis 1996. Vol. 36(2), P. 159178.

78. Várhegyi G., Antal M.J., Jakab E., Szabó P. Kinetic modeling of biomass pyrolysis. Journal of Analytical and Applied Pyrolysis 1997. Vol. 42(1), P. 73-87. 